\title{
Oculomotor Nerve Palsy as a Rare Presentation of Large B-Cell Central Nervous System Lymphoma
}

\author{
Hossein Golriz ${ }^{1,2}$, Shirin Haghighat ${ }^{3}$, Vahid Reza Ostovan ${ }^{1,2}$, Nasrin Shayanfar ${ }^{4}$, Mansour Parvaresh ${ }^{5}$, \\ Haniyeh Javanmardi ${ }^{1,6}$, Anahid Safari ${ }^{7}$ and Afshin Borhani-Haghighi ${ }^{1,2,{ }^{*}}$ \\ ${ }^{1}$ Clinical Neurology Research Center, Shiraz University of Medical Sciences, Shiraz, Iran \\ ${ }^{2}$ Department of Neurology, School of Medicine, Shiraz University of Medical Sciences Shiraz, Iran \\ ${ }^{3}$ Department of Hematology, Hematology Research Center, Medical Oncology and Stem Cell Transplantation, Shiraz University of Medical Sciences, Shiraz, Iran \\ ${ }^{4}$ Department of Pathology, Iran University of Medical Sciences, Tehran, Iran \\ ${ }^{5}$ Department of Neurosurgery, Iran University of Medical Sciences, Tehran, Iran \\ ${ }^{6}$ Student Research Committee, Shiraz University of Medical Sciences, Shiraz, Iran \\ ${ }^{7}$ Stem Cells Technology Research Center, Shiraz University of Medical Sciences, Shiraz, Iran \\ "Corresponding author: Clinical Neurology Research Center, Mohammad Rasulallah Research Tower, Khalili st, 10 th Floor, Shiraz, Iran. Tel/Fax:+98-7136281572, Email: \\ neuro.ab@gmail.com
}

Received 2019 October 12; Revised 2020 April 15; Accepted 2020 April 20.

Keywords: Brain Neoplasm, PCNSL, Lymphoma, Oculomotor Nerve Palsy

\section{Dear Editor,}

Primary central nervous system lymphoma (PCNSL) is a rare brain neoplasm with a very poor prognosis and limited treatment options (1). These malignant tumors include about $2 \%$ of all primary brain neoplasms (2). The treatment of choice in patients with PCNSL is systemic chemotherapy with high-dose methotrexate with or without whole-brain radiotherapy or intrathecal chemotherapy (3). Commonly, patients with PCNSL present with headache, seizure, vertigo, and mental/behavioral changes at the first visit (1). Focal neurological symptoms, cranial nerve palsy, cranial nerve symptoms, and neuropsychiatric symptoms are the other common presentations of PCNSL $(1,2)$. In this letter, we report a patient with large B-cell lymphoma, presenting with the manifestations of third nerve palsy.

A 29-year-old man referred with a headache, nausea/vomiting, diplopia, and left-sided ptosis two days before his admission to Namazi hospital affiliated to Shiraz University of Medical Sciences, Iran. He had a history of repeated nausea, vomiting, and weight loss for a year. The general physical examination was unremarkable, but he showed left-sided oculomotor nerve palsy in the neurological examination. The pupil size and reaction were normal. Other cranial nerves, mental status, sensorimotor and cerebellar functions, and meningeal signs were within normal limits.

Routine work-ups were unremarkable. Tumor markers were negative.
The laboratory tests for vasculitides were negative.

Anti-human immunodefeciency virus (HIV) antibody, HBs-antigen, and anti-hepatitis C virus (HCV) antibody were also negative. The serological assessment for the Epstein-Barr virus was negative, too. anti-acetylcholine receptor (anti-AchR) and anti-muscle specific kinase (antiMuSK) antibodies were negative. The CSF analysis showed WBC $79 / \mathrm{mm}^{3}$ (polymorphonuclear $45 \%$ and lymphocyte $34 \%$ ), glucose $20 \mathrm{mg} / \mathrm{dL}$, protein $104 \mathrm{mg} / \mathrm{dL}$, and LDH 90 $\mathrm{U} / \mathrm{L}$. The CSF culture and cytology were negative. Electromyography and repeated nerve stimulation tests were normal. Brain MRI showed a right-sided enhancing lesion adjacent to the pontine part of the fourth ventricle (Figure $1 \mathrm{~A}$ ), and thickening of the cisternal part of the left oculomotor nerve associated with contrast enhancement (Figure 1). Paranasal sinuses were normal in computerized tomography (CT). Brain CT angiography showed no aneurysm. Chest, abdominal, and pelvic CT scans were tumor-free. Ultimately, the patient was considered as a case of oculomotor neuroma and discharged with valproate, amitryptiline, and ondansetron.

After discharge, he visited an infectious disease specialist and received rifampin, doxycycline, and prednisolone with the impression of Neuro-Brucellosis. His ptosis disappeared, but he developed vertigo three months later. He was re-admitted to the hospital due to refractory vertigo, headache, nausea, vomiting, and diplopia. New MRI revealed several new intraventricular enhancing nodules in the frontal horn of the left lateral ventricle and the supe- 

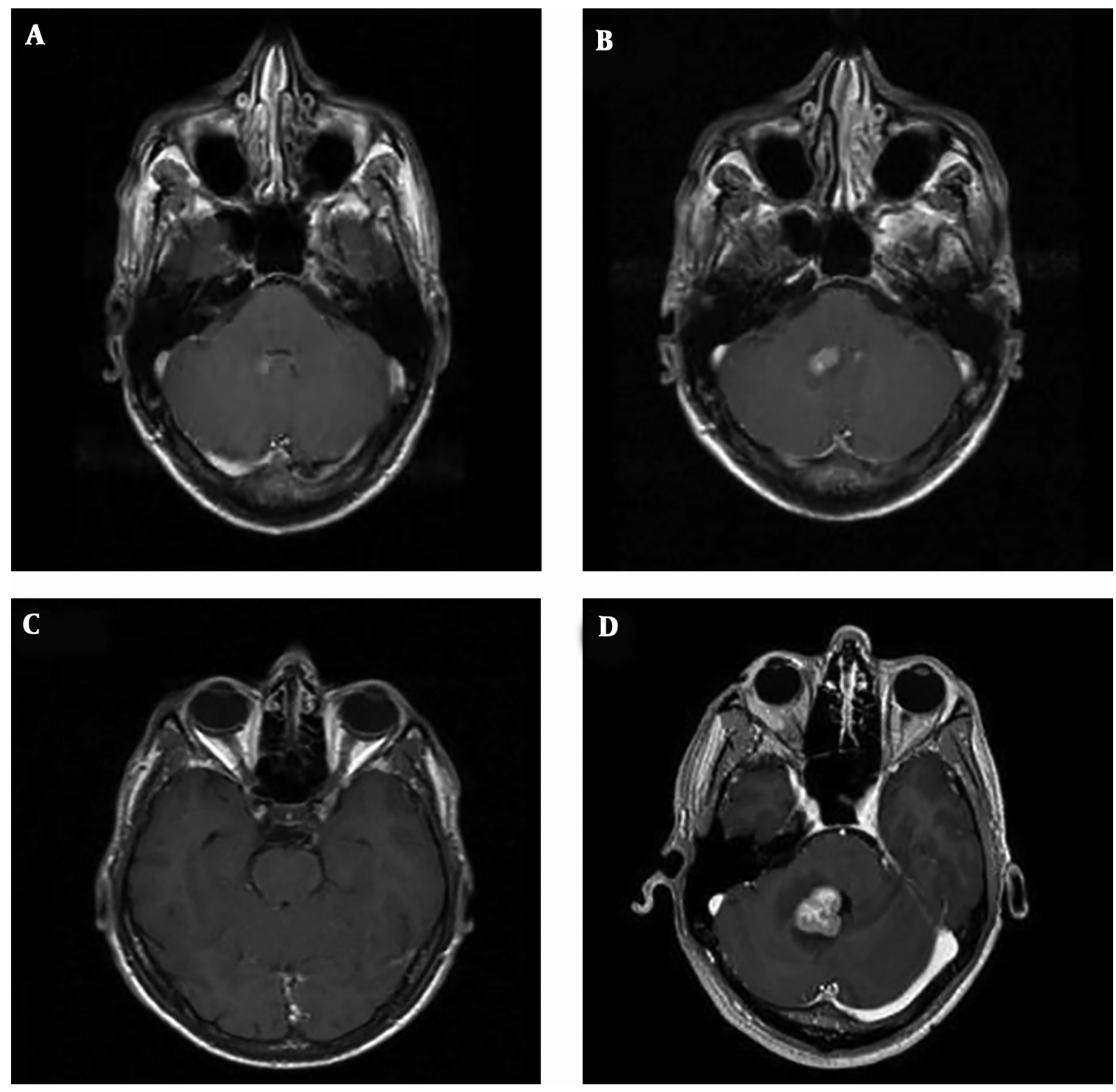

Figure 1. A, Contrast-enhanced T1-images (TR/TE: $350-550 / 8.3-9.5$ with $0.1 \mathrm{mmol} / \mathrm{kg} \mathrm{Gad}$ ) showing an enlarging lesion in the inferior aspect of the fourth ventricle in months 0 $\mathrm{B}$ and $\mathrm{C}$, three; and $\mathrm{D}$, five after developing third nerve palsy.

rior aspect of the fourth ventricle. The previously mentioned right paraventricular lesions and left cisternal lesions were enlarged (Figure $1 \mathrm{~B}$ and $\mathrm{C}$ ). Another CSF study showed five cells (all lymphocyte), protein $81 \mathrm{mg} / \mathrm{dL}$, glucose $4 \mathrm{mg} / \mathrm{dL}$ (concomitant blood sugar $119 \mathrm{mg} / \mathrm{dL}$ ), lactate dehydrogenase $122 \mathrm{U} / \mathrm{L}$, and lactate $65 \mathrm{mmol} / \mathrm{L}$. The CSF polymerase chain reactions for tuberculosis and brucellosis were negative. Despite negative CSF cytology, carcinomatous meningitis was considered an ongoing diagnosis. A whole-body scan indicated two focal active bone lesions in the left frontal and right occipital bones, which were in favor of tumoral involvement. Two months later, the rightsided lesion adjacent to the fourth ventricle became large enough for MRI-guided stereotactic biopsy (Figure 1D). The biopsy showed CD20 positive atypical lymphoid cells indicating diffused large B-cell lymphoma (Figure 2A and B).

Further investigations included CT of chest, abdomen, and pelvis, bone marrow aspiration, and biopsy, but the ophthalmic evaluation did not reveal lymphomatous involvement. After that, the patient received a combination 

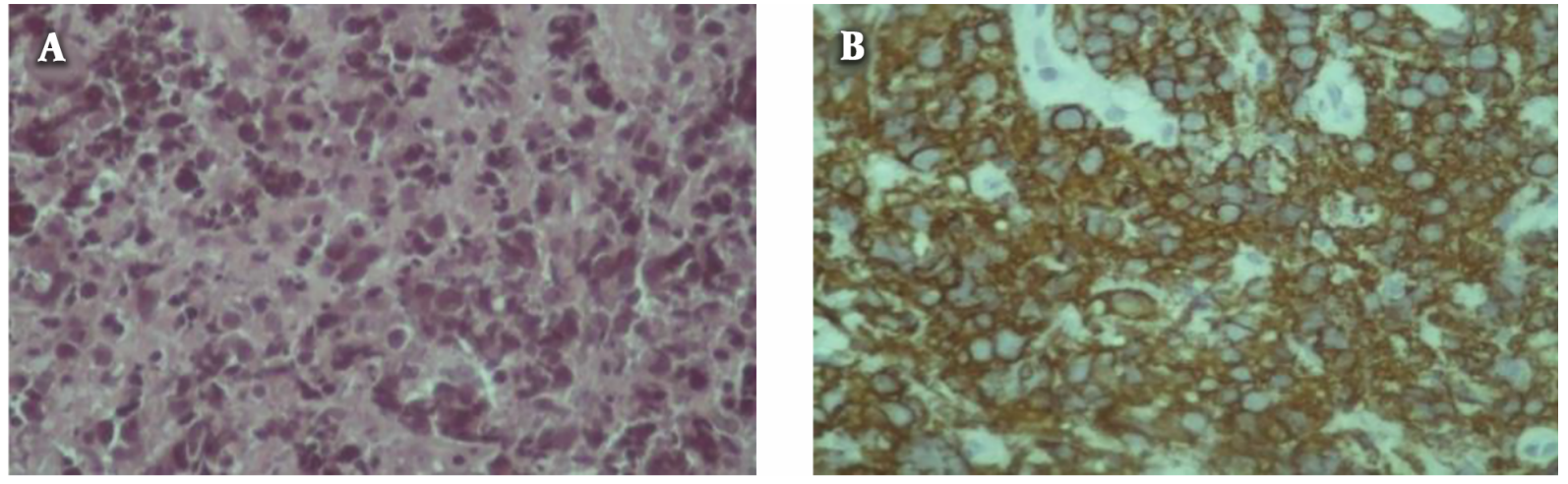

Figure 2. A, Infiltration of atypical lymphoid cells in the brain parenchyma (H \& E, 40×); B, diffuse membranous and cytoplasmic positivity of infiltrating lymphocytes for CD20 (IHC, $40 \times$ )

of chemotherapy agents consisting of six 14-day cycles of R-MVP (rituximab, methotrexate, procarbazine, and vincristine). The patient completed all chemotherapy cycles without any major toxicity with complete resolution of symptoms. Since postchemotherapy brain MRI revealed partial response, the patient was sent for whole-brain radiotherapy, which showed the complete resolution of brain lesions. Then, the patient was sent to receive consolidation with high-dose chemotherapy, followed by autologous stem cell rescue.

Primary central nervous system lymphoma (PCNSL) is a rare form of non-Hodgkin lymphoma with a poor prognosis, which is confined to the CNS. Patients with PCNSL mostly complain of headaches, seizures, vertigo, and mental status changes. Focal neurological deficits, cranial nerve symptoms, and neuropsychiatric symptoms are the other presentations of PCNSL (1). Rarely, PCNSL presents systemic manifestations such as fever, weight loss, and night sweating. The most common lesion sites are deep gray nuclei, periventricular white matter, corpus callosum, and CSF spaces $(4,5)$. Less common sites include the brainstem, cranial nerves, cavernous sinuses, and pineal and pituitary glands. The pattern of the intracranial distribution of the lesion in our case was inconsistent with the tendency of PCNSL to involve basal ganglia and periventricular white matter. In this case, the lesion involved the intraventricular area of the fourth ventricle and the cisternal portion of the left third cranial nerve.

Oculomotor nerve palsy commonly occurs due to leptomeningeal lymphoma that compresses the oculomotor nerve. This nerve exits in the midbrain through the interpeduncular cistern. Irregular contrast enhancement in the interpeduncular cistern is evident in brain MRI of patients with oculomotor nerve compression. Although the involvement of cranial nerves and their leptomeninges is commonly reported in many cases, isolated oculomotor palsy is unusual as the initial presentation of PCNSL. There are only a few case reports of oculomotor nerve palsy as the presenting manifestation of lymphoma $(6,7)$. Taga et al. (8) considered some "red falgs" for suspecting to lymphoma as the cause of third nerve palsy. These red flags included young age, progressive course, good response to corticosteroids, and relapse after stopping corticosteroids. The definite diagnosis of PCNSL is based on the histopathological confirmation in the absence of systemic lymphoma. Prompt diagnosis and management of patients are required to improve the survival outcome of patients diagnosed with PCNSL. This case report reconfirms considering PCNSL in the differential diagnosis of third nerve palsy.

\section{Footnotes}

Authors' Contribution: Afshin Borhani-Haghighi did conceptualization, visualization, data curation, writing the original draft, reviewing, and editing. Hossein Golriz, Shirin Haghighat, Vahidreza Ostovan, Nasrin Shayanfar, Mansour Parvaresh, Haniyeh Javanmardi, and Anahid Safari did data curation, writing the original draft, reviewing, and editing.

Conflict of Interests: The authors have no conflict of interest.

Funding/Support: The authors received no specific funding for this work.

Informed Consent: Informed consent was obtained for this research. 


\section{References}

1. Tan T, Rosa CSL, Han TF. Primary central nervous system lymphoma presenting as isolated oculomotor nerve palsy. Interdiscip Neurosurg. 2014;1(3):56-8. doi: 10.1016/j.inat.2014.06.003.

2. Bellinzona M, Roser F, Ostertag H, Gaab RM, Saini M. Surgical remova of primary central nervous system lymphomas (PCNSL) presenting as space occupying lesions: A series of 33 cases. Eur J Surg Oncol. 2005;31(1):100-5. doi: 10.1016/j.ejso.2004.10.002. [PubMed:15642434].

3. Grommes C, Rubenstein JL, DeAngelis LM, Ferreri AJM, Batchelor TT. Comprehensive approach to diagnosis and treatment of newly diagnosed primary CNS lymphoma. Neuro Oncol. 2019;21(3):296-305. doi: 10.1093/neuonc/noy192. [PubMed: 30418592]. [PubMed Central: PMC6380418].

4. Mansour A, Qandeel M, Abdel-Razeq H, Abu Ali HA. MR imaging features of intracranial primary CNS lymphoma in immune compe- tent patients. Cancer Imaging. 2014;14:22. doi: 10.1186/1470-7330-14-22. [PubMed: 25608570]. [PubMed Central: PMC4331827].

5. Mohile NA, Abrey LE. Primary central nervous system lymphoma. Semin Radiat Oncol. 2007;17(3):223-9. doi: 10.1016/j.semradonc.2007.02.008. [PubMed:17591569].

6. Heckmann JG. Oculomotor nerve palsy and diffuse large B cell lymphoma. Acta Neurol Belg. 2017;117(3):743-4. doi: 10.1007/s13760-017 0759-x. [PubMed: 28220398].

7. Tsai CH, Lee EJ, Liu YS, Chen YC, Shih YH, Chuang MT. Isolated oculomotor nerve palsy caused by diffuse large B cell lymphoma. Acta Neurol Belg. 2013;113(1):103-4. doi:10.1007/s13760-012-0100-7. [PubMed: 22729834].

8. Taga A, Russo M, Florindo I, Pavesi G. Isolated third cranial nerve palsy leading to the diagnosis of disseminated burkitt lymphoma: A CASE REPORT AND LITERATURE REVIEW. Neurologist. 2017;22(5):182-5. doi: 10.1097/NRL.0000000000000130. [PubMed: 28859023]. 